\title{
HOMOLOGOUSLY COLLAPSING STELLAR CORES ${ }^{1}$
}

\author{
Peter Goldreich \\ Division of Geological and Planetary Sciences, California Institute of Technology \\ AND \\ STEPHEN V. Weber \\ W. K. Kellogg Radiation Laboratory, California Institute of Technology \\ Received 1979 September 26; accepted 1979 December 5
}

\begin{abstract}
We investigate the collapse of nonrotating gas spheres with a polytropic equation of state: $n$ $=3$, corresponding to $\gamma=4 / 3$. Such polytropes provide a reasonable approximation to collapsing stellar cores during the early phase before nuclear density is reached. We find a family of exact homologously collapsing configurations. Homologous collapse of the entire core is possible if the pressure at a given density is reduced by up to $3 \%$ from the value for a marginally stable static core. For a greater pressure reduction, an inner core can collapse homologously, the mass of which varies as the $3 / 2$ power of the reduced pressure at the onset of collapse. Linear perturbations of these homologously collapsing solutions are separable in space and time. Low order radial and nonradial modes are calculated, and it is found that all modes are essentially stable.
\end{abstract}

Subject headings: stars: collapsed - stars: interiors - stars: supernovae

\section{INTRODUCTION}

Most work on stellar core collapse in supernovae has employed numerical codes for spherically symmetric hydrodynamics. This approach to the hydrodynamics permits a detailed treatment of the remaining physics, such as nuclear reactions, energy transport, neutrino transport, and the equation of state. However, it can be instructive to disregard the details of the thermodynamics and consider a simple analytic equation of state. Many results may then be obtained analytically, the relationship between the input physics and the consequent behavior is more transparent, and it is possible to investigate behavior (such as departures from spherical symmetry) that is more difficult to examine with numerical hydrodynamics.

According to recent work on the evolution of supernova progenitors (see Paczynski 1971; Ikeuchi et al. 1971; Arnett 1977b; Barkat 1977), stars massive enough to ignite carbon nonexplosively all evolve to a very similar precollapse configuration. This consists of a hot degenerate iron core of some $1-3 M_{\odot}$, with overlying burning shells. The core can be roughly approximated by an $n=3$ polytrope (Van Riper 1978; Nadyozhin 1977). It becomes dynamically unstable when the mean adiabatic index, $\bar{\gamma}=\int_{V} \gamma P d V / \int_{V} P d V$, drops below $4 / 3$. This results from some combination of inverse $\beta$ decay, endothermic dissociation of iron, and general relativity. After these effects reduce the pressure and initiate collapse, the increasing density soon causes the core to become opaque to neutrinos so

\footnotetext{
${ }^{1}$ Supported in part by the National Science Foundation (AST7821453 and AST76-80801 A02).
}

that the subsequent collapse is nearly adiabatic (Arnett 1977a; Bethe et al. 1979; Saenz and Shapiro 1979).

These results indicate that an $n=3, \gamma=4 / 3$ polytrope would be a reasonably simple approximation to the equation of state. By this we mean

$$
P=\kappa \rho^{\gamma},
$$

where $P$ is the total pressure, $\rho$ is the density, $\gamma=4 / 3$ is the adiabatic index, and $\kappa$ is a constant both in space and in time. "Adiabatic" normally means that $\kappa$ is constant in time along any given streamline, but not necessarily constant from streamline to streamline. "Polytropic" normally means that $\kappa$ is constant in space for a hydrostatic configuration, and $\gamma$ is replaced by $1+1 / n$, with $n$ the polytropic index. For a normal polytrope, $\gamma=d \log P / d \log \rho$ does not necessarily equal $1+1 / n$, nor is it necessarily constant.

The numerical models indicate that the proposed equation of state is reasonable for the precollapse core since the core resembles an $n=3$ polytrope. The mean $\gamma$ is $4 / 3$ at the onset of collapse, although the local value will range above and below this. The pressure reduction at the onset of collapse can be modeled by using a smaller $\kappa$ for collapsing cores than the hydrostatic cores. The statement that core collapse is adiabatic means in this case that it conserves entropy, not that the equation of state is as simple as equation (1). The equation of state will become stiffer at nuclear densities, an essential feature for causing a core bounce and for allowing a neutron star to form. However, the proposed equation of state should be reasonable at densities lower than nuclear density. This model is meant only to describe the early stages of collapse before nuclear density is reached. 
One nice feature of this equation of state is that the characteristic mass corresponding to the Jeans length is independent of density. Put another way, the gravitational energy and the thermal energy scale as the same power of the radius for homologous deformations of the star. Therefore, the center of the core should not run away from the outer parts during collapse, since the center alone would be less than the Jeans mass, regardless of the density increase. This suggests that a homologously collapsing solution could exist. Indeed, homologous collapse of the inner part of the core has been observed in the numerical models (Nadyozhin 1977; Epstein 1977; Arnett 1977a; Van Riper and Arnett 1978; Van Riper 1978). A more complicated equation of state would not give identical scaling of gravitational and thermal energy with core radius, so that homologous exact solutions for collapsing cores would not be expected. However, if the true equation of state is not too different from that given by equation (1), the departure from homology should be small.

The stability of core collapse, especially for nonradial modes, is an important question. One reason is that no gravitational radiation is emitted by spherically symmetric systems. Growth of nonradial modes is essential if supernovae are to be powerful sources of gravitational radiation. It is relatively easy to investigate the stability of homologously collapsing cores. Since the Jeans mass is independent of density in our model, there is no tendency toward fragmentation. This suggests that the collapse will be stable.

In $\S$ II, we determine that a family of homologous collapsing solutions does exist and discuss the properties of these solutions. The equations describing linear perturbations of those configurations are derived in $\S$ III, and the normal modes are discussed in $\S$ IV. Section V presents a summary and conclusions.

\section{HOMOLOGOUSLY COLLAPSING CORES}

We begin with the equation of continuity, Euler's equation, and Poisson's equation:

$$
\begin{gathered}
\frac{\partial \rho}{\partial t}+\nabla \cdot(\rho \boldsymbol{u})=0 \\
\frac{\partial \boldsymbol{u}}{\partial t}+\nabla\left(\frac{1}{2}|\boldsymbol{u}|^{2}\right)+(\nabla \times \boldsymbol{u}) \times \boldsymbol{u}+\nabla h+\nabla \phi=0 \\
\nabla^{2} \phi-4 \pi G \rho=0
\end{gathered}
$$

Here, $\boldsymbol{u}$ is the fluid velocity; $\phi$ is the gravitational potential; $G$ is the gravitational constant; and $h$ is the heat function $\left(h=\int d P / \rho=4 \kappa \rho^{1 / 3}\right.$ for our equation of state). If the flow is vorticity free, the velocity may be obtained from a stream function: $u=\nabla v$. (Note that an arbitrary constant can be added to $v$.) Then, equation (3) can be integrated

$$
\frac{\partial v}{\partial t}+\frac{1}{2}|\nabla v|^{2}+h+\phi=0 .
$$

The constant of integration has been incorporated into the potential.

It is conventional for polytropes to use a radial scale corresponding to the Jeans length (see Eddington 1926). The scale factor is time dependent in our case:

$$
a(t)=\left(\gamma P_{c} / \rho_{c}\right)^{1 / 2} /\left(\pi \gamma G \rho_{c}\right)^{1 / 2}=\rho_{c}^{-1 / 3}\left(\frac{\kappa}{\pi G}\right)^{1 / 2},
$$

where the subscript $c$ indicates the value at $R=0$. We scale the radial coordinate from (dimensioned) $\boldsymbol{R}$ to (dimensionless) $\boldsymbol{r}=\boldsymbol{R} / a(t)$. The differential equations are transformed by this time dependent scaling to

$$
\begin{gathered}
\frac{1}{\rho} \frac{\partial \rho}{\partial t}+a^{-1}\left(a^{-1} \nabla v-\dot{a} \boldsymbol{r}\right) \cdot \nabla \rho / \rho+a^{-2} \nabla^{2} v=0, \\
\frac{\partial v}{\partial t}-\frac{\dot{a}}{a} \boldsymbol{r} \cdot \nabla v+\frac{1}{2} a^{-2}|\nabla v|^{2}+h+\phi=0, \\
a^{-2} \nabla^{2} \phi-4 \pi G \rho=0,
\end{gathered}
$$

where we have also substituted the stream function into the continuity equation. Next, we scale the density in terms of the central density,

$$
\rho=\rho_{c} f^{3}=\left(\frac{\kappa}{\pi G}\right)^{3 / 2} a^{-3} f^{3},
$$

and the potential in terms of the square of the central sound speed,

$$
\phi=\left(\frac{\gamma P_{c}}{\rho_{c}}\right) \psi=\frac{4}{3}\left(\frac{\kappa^{3}}{\pi G}\right)^{1 / 2} \frac{\psi}{a} .
$$

To seek a homologous solution, we set $v=1 / 2 a \dot{a} r^{2}$ or $\boldsymbol{u}=\dot{a} \boldsymbol{r}$ so that the continuity equation reduces to the trivial relation $\dot{f}=0$, which states that the density profile does not evolve. Euler's equation yields

$$
(f+\psi / 3) / r^{2}=-\frac{1}{8}\left(\frac{\pi G}{\kappa^{3}}\right)^{1 / 2} a^{2} \ddot{a}=\lambda / 6,
$$

which is separable in $\boldsymbol{r}$ and $t$. The expression on the left is a function of radius only, while the middle expression depends on time only, so both have been equated to a constant. This pair of equations implies

$$
\psi=\frac{1}{2} \lambda r^{2}-3 f
$$

and also gives a nonlinear differential equation for $a(t)$. The general solution to this differential equation gives the time dependence of the most general exactly homologous solution.

The differential equation for $a(t)$ can be integrated once, yielding the energy integral

$$
\frac{1}{2} \dot{a}^{2}=\frac{4}{3} \lambda\left(\frac{\kappa^{3}}{\pi G}\right)^{1 / 2} a^{-1}+C
$$

The constant of integration, $C$, determines the contraction (or expansion) velocity when the radius of the star is infinite. Equation (14) can be integrated again if $C=0$ and $\lambda>0$ :

$$
a=(6 \lambda)^{1 / 3}\left(\frac{\kappa^{3}}{\pi G}\right)^{1 / 6}\left(t+t_{0}\right)^{2 / 3}
$$


The second constant of integration, $t_{0}$, is the time at which the radius goes to zero. We shall henceforth assume $t_{0}=0$. In a realistic case of core collapse, $C$ $\neq 0$ because $\dot{a}=0$ at some finite $a$. However, the value of $C$ should have little effect on the solution for $a(t)$ as $a \rightarrow 0$. Indeed, we shall see in $\S$ IV that homologous linear perturbations of the solution given by equation (15) go to zero as $a \rightarrow 0$.

For $\lambda=0$, the star has zero binding energy and is neutrally stable to homologous radial perturbations. The most general solution for $a(t)$ in this special case is a linear function of time. However, we shall discuss only the static $\lambda=0$ core, and shall not consider the core with $\dot{a}=$ constant $\neq 0$.

We use equation (13) to eliminate $\psi$ in Poisson's equation, and obtain an ordinary differential equation for the radial structure

$$
\frac{1}{r^{2}} \frac{d}{d r}\left(r^{2} \frac{d f}{d r}\right)+f^{3}=\lambda
$$

This reduces to the Lane-Emden equation for polytropic index $n=3$ (Eddington 1926) in the limit $\lambda=0$. The appropriate boundary conditions are $f(0)=1$, $d f / d r(0)=0$.

Numerical solutions of equation (16) for several values of $\lambda$ are illustrated in Figure 1 . The case $\lambda=0$ is the usual $n=3$ polytrope. Negative values of $\lambda$ are not shown or discussed because they give unbound systems which cannot collapse and have a different time dependence than that given by equation (15). It can be seen that the solutions for positive $\lambda$ differ little in the inner parts, but that the outer layers become more extended as $\lambda$ increases

There is a maximum value of $\lambda$ for which a physical solution is possible, $\lambda_{m} \approx 0.00654376$. Mathematically, this limit exists because $f(r)$ becomes tangent to $f=0$ at the outer boundary in the limiting case, whereas for $\lambda>\lambda_{m}, f$ remains finite at all $r$. Physically, the limiting value of $\lambda$ is reached when the surface of

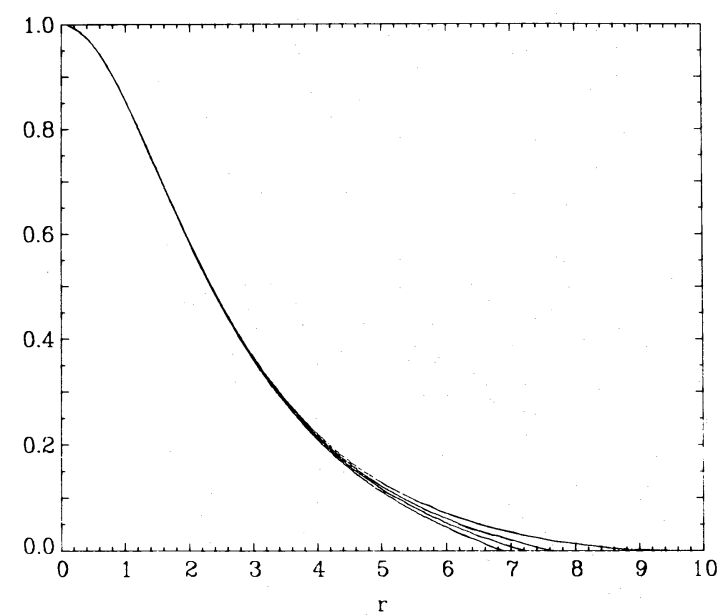

FIG. 1.-Density profile $f(r) \propto \rho^{1 / 3}$ of homologously-collapsing polytropes, for (left to right) $\lambda=0,0.002,0.004$, and $\lambda_{m}$. the core is in free fall. The condition $\dot{u}\left(R_{s}\right)=$ $-G M / R_{s}{ }^{2}$ becomes $\bar{\rho} / \rho_{c}=\lambda$ in dimensionless units, after equation (12) is used to evaluate the acceleration, and the core mass, $M$, is expressed in terms of the mean density, $\bar{\rho}$. In order to see that $\bar{\rho} / \rho_{c}=\lambda$ when $\lambda=\lambda_{m}$, we write

$$
\bar{\rho} / \rho_{c}=\left(4 \pi r_{s}^{3} / 3\right)^{-1} \int_{0}^{r_{s}} 4 \pi r^{2} f^{3} d r,
$$

and use equation (16) to evaluate $f^{3}$. The integral then can be performed, yielding

$$
\bar{\rho} / \rho_{c}=\lambda-\left(3 / r_{s}\right) d f\left(r_{s}\right) / d r .
$$

Thus, the condition for free fall at the surface can be seen to be satisfied when $d f\left(r_{s}\right) / d r=0$.

The core mass for the collapsing polytropes is $M$ $=4 / 3 \pi r_{s}{ }^{3}\left(\bar{\rho} / \rho_{c}\right)(\kappa / \pi G)^{3 / 2}$. As $\lambda$ increases from zero to $\lambda_{m}, r_{s}$ increases from approximately 6.897 to 9.889 , while $\bar{\rho} / \rho_{c}$ decreases from 0.01846 to 0.006544 . The product $r_{s}{ }^{3} \bar{\rho} / \rho_{c}$ is relatively insensitive to $\lambda$, increasing only by a factor 1.0449 in this interval. Specifying $M$ and $\lambda$ determines $\kappa$, and the range of $\lambda$ corresponds to a range of $2.9 \%$ in $\kappa$. Consequently, if we begin with a static $(\lambda=0)$ core, we can reduce $\kappa$, and thus the pressure, by no more than $2.9 \%$, and still find a homologously collapsing configuration for the entire core. The reason for this small range is that the static configuration is only neutrally stable and is highly centrally condensed.

The pressure reduction initiating collapse in supernovae is substantially larger (Bethe et al. [1979] obtain $26 \%$ ). Although the entire core cannot collapse homologously when the pressure reduction is this large, a less massive inner core can do so, while the remainder of the core is left behind. The $\lambda=\lambda_{m}$ homologous solution should be a good approximation to the structure of the inner core to the extent that the pressure due to the material further out (which is nearly in free fall) is negligible. The mass of the inner core will be

$$
M_{\text {ic }}=1.0449\left(\frac{\kappa}{\kappa_{0}}\right)^{3 / 2} M_{0},
$$

where $M_{0}$ and $\kappa_{0}$ are the mass and the value of $\kappa$ for the marginally stable initial static core.

This prediction for the mass of the homologous inner core is in good agreement with the results of Van Riper (1978), who numerically integrates collapses, starting from an $n=3$ polytrope, with $\gamma=1.32$. His core masses agree with the prediction of equation (17) to within a few percent for pressure reductions of up to $50 \%$. His inner core mass is $30 \%$ larger than our prediction when the pressure reduction is $70 \%$, but this difference is only $6 \%$ of the total core mass and may not be well determined. Note, however, that Van Riper finds that the mass of the homologous inner core is sensitive to the value of $\gamma$, so that our quantitative predictions may be in error if $\gamma$ differs substantially from $4 / 3$. 
III. LINEAR PERTURBATION EQUATIONS

Consider linear perturbations of the homologously collapsing configurations described in the preceding section. The perturbed dynamical variables are written as

$$
\begin{gathered}
v(r, t)=\frac{1}{2} a \dot{a} r^{2}+\frac{a^{2}}{t_{f f}} v_{1}(r) g(t), \\
\rho(r, t)=\left(\frac{\kappa}{\pi G}\right)^{3 / 2} a^{-3} f^{3}\left[1+\rho_{1}(r) g(t)\right], \\
\phi(r, t)=\frac{4}{3}\left(\frac{\kappa^{3}}{\pi G}\right)^{1 / 2} a^{-1}\left[\psi_{0}+\psi_{1}(r) g(t)\right] .
\end{gathered}
$$

The function $g(t)$ gives the time dependence of the scaled perturbations, and is assumed to have the form,

$$
\begin{aligned}
g(t)=\exp \left[p \int^{t} t_{f f}^{-1} d t^{\prime}\right] & =\exp \left(p t / t_{f f}\right), \quad \lambda=0, \\
& =t^{(2 / 9 \lambda)^{1 / 2} p,} \quad \lambda \neq 0,
\end{aligned}
$$

where

$$
\begin{aligned}
t_{f f} & =\left(\frac{4}{3} \pi G \rho_{c}\right)^{-1 / 2} & & (\text { for all } \lambda), \\
& =(9 \lambda / 2)^{1 / 2} t & & (\text { for } \lambda \neq 0) .
\end{aligned}
$$

We have chosen to work with perturbations of the stream function rather than to work with the displacement vector as is more conventional (see, e.g., Eddington 1926; Ledoux and Walraven 1958). It is algebraically simpler to work with scalar variables, especially in an accelerated coordinate system.

Linearizing equations (7)-(9), and using equations (18)-(20) to define the perturbations, we obtain

$$
\begin{aligned}
\nabla^{2} v_{1}+3 \nabla \log f \cdot \nabla v_{1}+p \rho_{1} & =0 \\
{\left[p+(\lambda / 2)^{1 / 2}\right] v_{1}+f \rho_{1}+\psi_{1} } & =0 \\
\nabla^{2} \psi_{1}+3 f^{3} \rho_{1} & =0
\end{aligned}
$$

It can be seen that the time dependence due to the collapse of the unperturbed configuration has cancelled out of the dynamical equations, leaving an eigenvalue problem.

Cowling (1941) classified the modes of nonradial oscillation of a spherical star. The $g$-modes have a displacement vector which is primarily horizontal. They produce little pressure perturbation, and gravity is the principal restoring force. For the $p$-modes, the displacement vector is primarily radial, and pressure is the dominant restoring force. Cowling also identifies a fundamental or $f$-mode, for which the radial component of the displacement vector and the pressure perturbation are both constant in sign along any given radius vector. The $f$-mode is intermediate in frequency between the $p$-modes and the $g$-modes. The frequency for $p$-modes increases as the number of modes increases, while the frequency decreases with increasing order for $g$-modes. We shall not distinguish the $f$ mode, but shall regard it as the lowest order $p$-mode.
Cowling (1941) showed that for $\gamma=1+1 / n$, which is assumed in our model, the $g$-modes are just neutral convective modes. These have $p=0, \rho_{1}(r)=\psi_{1}(r)=$ $0, \nabla^{2} v_{1}+3 \nabla \log f \cdot \nabla v_{1}=0$. We shall not discuss the $g$-modes further. For the $p$-modes, equation (24) can be used to eliminate $\rho_{1}$. Assuming that the angular dependence of the perturbations is given by spherical harmonics, we obtain two second-order ordinary homogeneous differential equations

$$
\begin{gathered}
\frac{f}{r^{2}} \frac{d}{d r}\left(r^{2} \frac{d w}{d r}\right)-\frac{l(l+1)}{r^{2}} f w+3 \frac{d f}{d r} \frac{d w}{d r}-\psi_{1}+m w=0, \\
\frac{1}{r^{2}} \frac{d}{d r}\left(r^{2} \frac{d \psi_{1}}{d r}\right)-\frac{l(l+1)}{r^{2}} \psi_{1}+3 f^{2} \psi_{1}-3 f^{2} m w=0,
\end{gathered}
$$

where $v_{1}=p w$ and $m=-p\left[p+(\lambda / 2)^{1 / 2}\right]$. Thus, we have $p=(\lambda / 8)^{1 / 2} \pm(\lambda / 8-m)^{1 / 2}$, and $g(t)$ becomes $\exp \left( \pm \mathrm{im}^{1 / 2} t / t_{f f}\right)$ for $\lambda=0 ; t^{q} \propto a^{3 q / 2}, q=-1 / 6 \pm$ $(1 / 36-2 m / 9 \lambda)^{1 / 2}$ for $\lambda \neq 0$. The displacement vector $\xi=\delta \boldsymbol{r} / r$ is related to $w$ by $\xi=\nabla w$.

It can easily be proved that the eigenvalues, $m$, are real and that eigenfunctions $\xi_{1}(\boldsymbol{r}), \xi_{2}(\boldsymbol{r})$ corresponding to different eigenvalues $m_{1} \neq m_{2}$ are orthogonal: $\int_{V} f^{3} \xi_{1} \cdot \xi_{2} d V=0$.

The boundary conditions for differential equations (26) and (27) are regularity conditions. Regularity at the origin for $w$ and $\psi_{1}$ is insured by $w, \psi_{1} \propto r^{l}$ as $r \rightarrow 0$. Regularity of $w$ at the surface requires $3(d f / d r)(d w / d r)$ $-\psi_{1}+m w=0$ at $r=r_{s}$. For $l \neq 0$, regularity of $\psi_{1}$ at infinity is insured by $\psi_{1} \propto r^{-(l+1)}$ at $r=r_{s}$ [i.e., $d \psi_{1} / d r$ $\left.+(l+1) \psi_{1} / r=0\right]$. For $l=0$, the fourth boundary condition serves only to determine the arbitrary constant in the definition of the stream function, and any convenient linearly independent condition can be specified.

\section{NORMAL MODES}

We have found eigenvalues and eigenfunctions of equations (26) and (27) numerically, using the method of inverse iteration (Wilkinson 1955). Eigenvalues $(m)$ for the lowest three modes for $l=0,1,2$ and $0 \leq \lambda$ $\leq \lambda_{m}$ are plotted in Figure 2. Some of the modes for the static $\lambda=0$ case have been computed before. Schwarzschild (1941) has evaluated radial modes, and our results are in satisfactory agreement with his. For $l$ $=\lambda=0$, it is possible to reduce the system of equations to a single second-order differential equation, but we have not done so because this is not possible in the general case. Cowling (1941) has obtained the eigenvalue for the lowest $l=2$ mode for $\lambda=0$. He approximates the potential perturbation rather than including it exactly; however, his eigenvalue is still in reasonable agreement with ours.

The lowest radial mode can be found analytically. It is $m=-3 \lambda, w=r^{2}, \psi_{1}=6[r(d f / d r)+f]-3 \lambda r^{2}$. This mode corresponds to a homologous perturbation of the entire core. Consequently, it perturbs the particular homologously collapsing solution we have used 


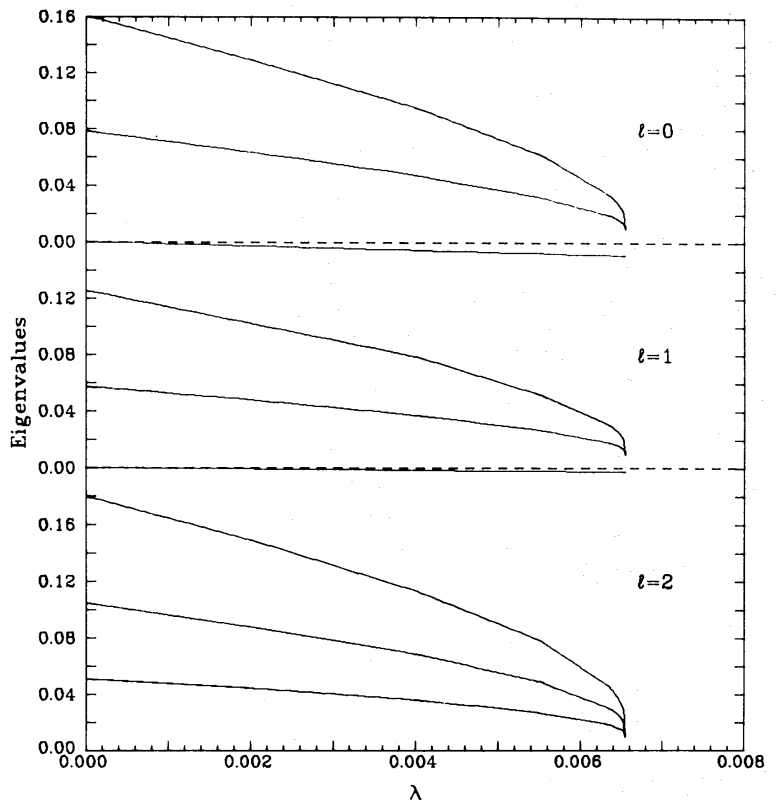

Fig. 2.-Eigenvalues $(m)$ as functions of $\lambda$ for $l=0,1,2$, for modes with (bottom to top) $0-2$ nodes.

for our unperturbed configuration into the general homologously-collapsing solution. The value of $m$ gives a time dependence of either $t^{-1}$ or $t^{2 / 3}$. The perturbations do grow in time for the former case, but this mode is produced simply by setting $t_{0} \neq 0$ in equation (15). This can be seen by

$$
\begin{aligned}
{\left[R\left(t+t_{0}\right)-R(t)\right] / R(t) } & \\
& \approx \dot{R}(t) t_{0} / R(t) \propto t^{-1 / 3} / t^{2 / 3}=t^{-1} .
\end{aligned}
$$

Thus, this should not be considered a growing mode. It is only an indication that one has chosen an inconvenient origin of time for the unperturbed configuration. The $t^{2 / 3}$ mode is a linearization of the $C \neq 0$ solution of equation (14); that is, the solution with a nonzero collapse velocity in the limit $a \rightarrow \infty$. The amplitude of this mode goes to zero as $t \rightarrow 0$, confirming our expectation that the value of $C$ becomes unimportant as $a \rightarrow 0$.

The lowest $l=1$ mode is a trivial solution, giving a uniform displacement of the entire star. This solution has $m=-\lambda$, giving a time dependence for $w$ and $\psi_{1}$ of $t^{-2 / 3}$ or $t^{1 / 3}$. More relevant is the time dependence of $\delta \boldsymbol{R}=\operatorname{ar} \boldsymbol{\nabla} w \propto t^{0}$ or $t^{1}$. These cases correspond to a neutral displacement or a constant translation velocity of the entire star. The eigenfunctions are $w=r, \psi_{1}$ $=3(d f / d r)-\lambda r$.

For all modes except the two special cases discussed above, the eigenvalue approaches the same limit $m$ $\rightarrow(25 / 8) \lambda_{m}$ as $\lambda \rightarrow \lambda_{m}$. The reason for this is not difficult to understand. First, if we employ the WKBJ approximation for short wavelength perturbations, we find that the radial wavenumber, $k$, varies as $k \propto(P / \rho)^{-1 / 2} \propto f^{-1 / 2}$. When both $f$ and its derivative go to zero at the surface, an infinite number of wavelengths will be found in any neighborhood of $r_{s}$. More rigorously, we can find an asymptotic solution for $\Delta r=r_{s}-r \ll 1, f(\Delta r) \propto \Delta r^{2}$. This solution is $w \propto \Delta r^{q}, q=-5 / 2 \pm(25 / 4-2 m / \lambda)^{1 / 2}$. We see that $q$ becomes imaginary for $m / \lambda>25 / 8$, producing an essential singularity at $\Delta r=0$. For $\lambda<\lambda_{m}, f(\Delta r)$ will become linear for $\Delta r$ sufficiently small, and $w(\Delta r)$ will become linear rather than oscillatory in that regime. Consequently, for eigenfunctions with a finite number of modes, $m \rightarrow 25 / 8 \lambda_{m}$ and the modes become concentrated to the surface as $\lambda \rightarrow \lambda_{m}$. The behavior of the eigenfunctions as $\lambda \rightarrow \lambda_{m}$ is illustrated by Figure 3 , which shows the $l=2$ eigenfunctions with two modes for a sequence of values of $\lambda$. The variation of the amplitude of $w(r)$ predicted by WKBJ theory, $w(r) \propto r^{-1} f^{-5 / 4}$, has been removed because the amplitude varies too much to permit a worthwhile plot of $w(r)$ by itself.

The singular behavior at $\lambda_{m}$ should be regarded as an artifact of the special mathematical character of this limiting solution. For a real stellar core there will be some finite pressure at the surface, although this will generally be much less than the central pressure. A finite sound speed at the surface will insure that the normal modes are regular and that the eigenfrequencies are isolated.

Figure 4 illustrates eigenfunctions for $\lambda=0, l=2$, and $0-3$ nodes, scaled as in Figure 3. These particular eigenfunctions are characteristic in their qualitative character. Modes for different $\lambda$ not too close to $\lambda_{m}$ look much the same, as do modes for different $l$, except for their behavior as $r \rightarrow 0$. The power in these modes is concentrated near the surface, as in $p$-modes generally. This feature is somewhat obscured in the figures by the scaling.

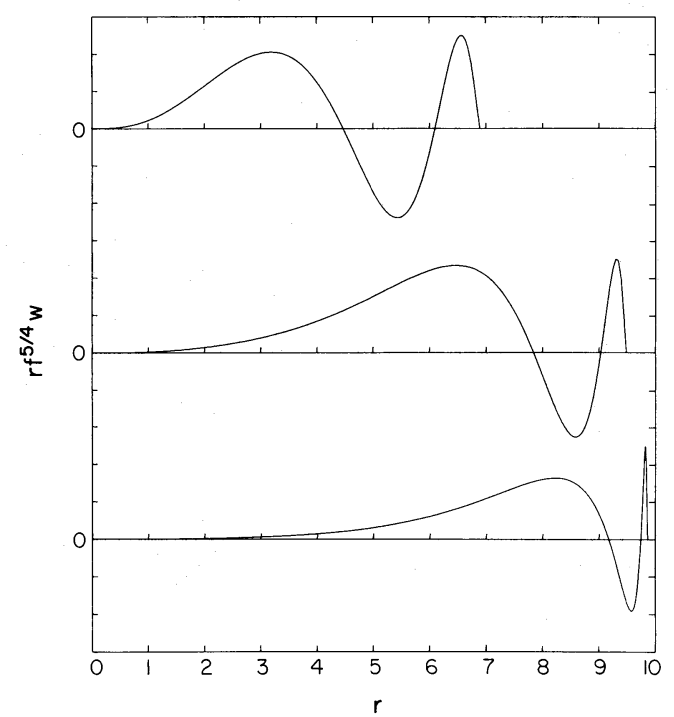

FIG. 3. - Scaled eigenfunctions $r f(r)^{5 / 4} w(r)$ for $l=2$, two nodes, and (top to bottom) $\lambda=0,0.0065$, and 0.0065435 . The vertical scale is arbitrary. Note how the nodes concentrate toward the surface as $\lambda \rightarrow \lambda_{m}$. 


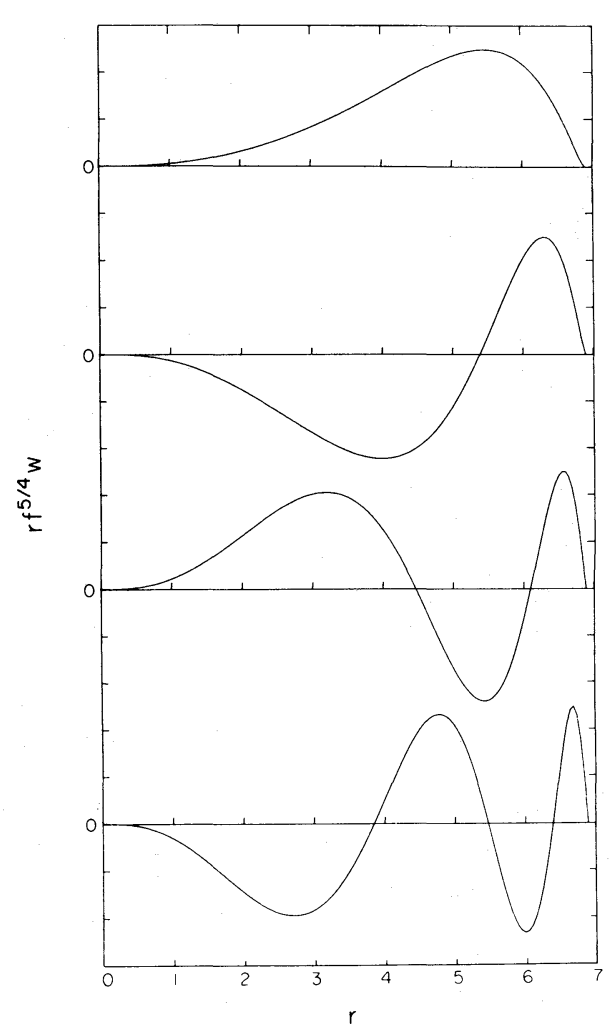

FIG. 4.-Scaled eigenfunctions $r f(r)^{5 / 4} w(r)$ for $l=2, \lambda=0$, and $0-3$ nodes. The vertical scale is arbitrary. Modes of different $l$ and $\lambda$ are qualitatively similar to these.

The most important characteristic of the normal modes is that all (excepting the two special cases discussed earlier) are oscillatory. The eigenvalues, $m$, are always greater than $25 \lambda / 8$. This limiting case gives a time dependence of $t^{q}, q=-1 / 6 \pm(2 / 3)^{1 / 2} i$. It is sufficient for $m / \lambda>1 / 8$ to obtain a complex eigenfrequency. The amplitude of the oscillations for all modes does increase during the collapse as $t^{-1 / 6} \propto a^{-1 / 4}$. This increase in amplitude corresponds to adiabatic amplification of sound waves due to compression. Amplification of this sort should not be described as instability. Also, the small exponent insures that perturbations will not attain high amplitude during collapse if they are initially small, unless the collapse is by a very large factor. Even in the collapse of a stellar core to a neutron star, in which the radius is reduced by a factor of order $10^{2-3}$, perturbations will be amplified only by a factor of 3-6.

\section{CONCLUSIONS}

Our polytropic models for collapsing stellar cores can explain the formation of a homologous inner core, which has been observed in numerical models. The mass of the inner core is given by the model: it is essentially the Chandrasekhar mass corresponding to the reduced entropy of the adiabatic phase of the collapse (to be precise, it is up to $4.5 \%$ larger than the Chandrasekhar mass). The density profile of the inner core should be similar to that of a stationary $n=3$ polytrope, but with somewhat more extended outer layers.

This homologously collapsing core is stable i1, the sense that perturbations are oscillatory, with the amplitude of the fractional displacement increasing as $R_{s}^{-1 / 4}$ as the core radius decreases. This may be contrasted with the pressure-free case, in which perturbations grow monotonically and rapidly (LyndenBell 1964; Lin, Mestel, and Shu 1965). The fact that pressure can stabilize spherical collapse is familiar, of course. Hunter (1962) discusses the stability of perturbations of homogeneous gas spheres with $\gamma=4 / 3$. In that case, the unperturbed collapse is effectively pressure-free because there is no pressure gradient, and perturbations are stable only for sufficiently short wavelengths. Lynden-Bell's (1979) statement based on the homogeneous case, that the shape of a sphere will be stable only if the inward acceleration of the boundary is less than $2 / 5$ of the free fall value, does not apply to our models. Our spheres are stable even in the limiting case when the surface collapses in free fall. However, the collapse of the interior is always slower than free fall.

Nonspherically symmetric collapses have been calculated numerically by Shapiro (1977) and Saenz and Shapiro $(1978,1979)$. These simplified models, employing homogeneous spheroids or ellipsoids, predict that departures from spherical symmetry and the emission of gravitational radiation become important only after the bounce of the core at nuclear densities. We concur that cores in which rotation is not important will remain very nearly spherically symmetric, and therefore will not emit significant gravitational radiation, before the core bounce.

The hydrodynamics of core collapse up until the core bounce can be understood in terms of this relatively simple analytic model. The interesting hydrodynamics of the bounce itself and the production of a reflected shock must be approached in another way.

We are grateful for comments by Doug Keeley and Frank Shu. We also acknowledge Glen Hermannsfeldt for excellent assistance with the computing. Stephen V. Weber was supported by a Chaim Weizmann postdoctoral fellowship. This work was supported by NSF grants AST78-21453 and AST76-80801 A02.

\section{REFERENCES}

Arnett, W. D. 1977a, Ap. J., 218, 815.

.1977b, Ap. J. Suppl., 35, 145.

Barkat, Z. 1977, in Supernovae, ed. D. N. Schramm (Dordrecht: Reidel), p. 131

Bethe, H. A., Brown, G. E., Applegate, J., and Lattimer, J. M. 1979, Nucl. Phys., A324, 487.
Cowling, T. G. 1941, M.N.R.A.S., 101, 367.

Eddington, A. S. 1926, The Internal Constitution of the Stars (Cambridge: Cambridge University Press).

Epstein, R. I. 1977, in Supernovae, ed. D. N. Schramm (Dordrecht: Reidel), p. 183.

Hunter, C. 1962, Ap. J., 136, 594. 
Ikeuchi, S., Nakazawa, K., Murai, T., and Hayashi, C. 1971, Prog. Theor. Phys., 46, 1713.

Ledoux, P., and Walraven, T. H. 1958, Handbuch der Physik, 51, 353.

Lin, C. C., Mestel, L., and Shu, F. H. 1965, Ap. J., 142, 1431.

Lynden-Bell, D. 1964, Ap. J., 139, 1195. 1979, Observatory, 99, 89.

Nadyozhin, D. K. 1977, Ap. Space Sci., 51, 283.

Paczynski, B. E. 1971, Acta Astr., 21, 271.
Saenz, R. A., and Shapiro, S. L. 1978, Ap. J., 221, 286. 1979, Ap. J., 229, 1107.

Schwarzschild, M. 1941, Ap. J., 94, 245

Shapiro, S. L. $1977, A p$. J., 214, 566.

Van Riper, K. A. 1978, Ap. J., 221, 304.

Van Riper, K. A., and Arnett, W. D. 1978, Ap. J. (Letters), 225, L129.

Wilkinson, J. H. 1955, The Algebraic Eigenvalue Problem (Oxford: Clarendon Press).

Note added in proof.-Van Riper (1980, private communication) has provided us with homologous core masses obtained by numerical integration of collapses, in which the equation of state (1) is employed. The homologous core masses agree with our analytic result (eq. [17]) to within the accuracy claimed for the numerical calculation $( \pm 2 \%)$, provided the pressure reduction initiating collapse is not too large. The numerical calculations give significantly larger homologous core masses than the analytic formula if $\kappa / \kappa_{0} \lesssim 0.2$. This can be explained easily. The gradients of density and pressure go to zero at the center of the initial configuration. A homogeneous pressure-free sphere collapses homologously, and a pressure gradient-free configuration is dynamically equivalent to one that is pressure-free. Therefore, the nearly homogeneous central part of the initial configuration will collapse nearly homologously, regardless of the equation of state, until the small initial gradients can produce an appreciable effect.

Our analytic results should always be valid asymptotically as the density goes to infinity. However, if the initial pressure reduction is very large, the homologous core must form from material that is initially nearly homogeneous, so the asymptotic solution may not be reached until the density has increased by a larger factor than a real core collapse would provide. Fortunately, the actual pressure reduction which initiates stellar core collapse is expected to be well within the regime in which our results should be valid.

Peter Goldreich: Division of Geological and Planetary Sciences, 170-25, California Institute of Technology, Pasadena, CA 91125

Stephen V. Weber: Theoretical Astrophysics, 130-33, California Institute of Technology, Pasadena, CA 91125 